\title{
Pathogenesis of the Adult Respiratory Distress Syndrome
}

\author{
EVIDENCE OF OXIDANT ACTIVITY IN \\ BRONCHOALVEOLAR LAVAGE FLUID
}

\author{
Charles G. Cochrane, Roger Spragg, and Susan D. Revak, Department \\ of Immunology, Scripps Clinic and Research Foundation, La Jolla, California \\ 92037; Pulmonary Division, University of California, San Diego, \\ La Jolla, California 92093
}

\begin{abstract}
A B S TRACT Evidence is presented indicating that oxidants are generated in lungs of patients with the adult respiratory distress syndrome (ARDS). The evidence was derived from observations that $\alpha$-1-PI, recovered in bronchoalveolar lavage (BAL) fluid, had been inactivated by oxidation, presumably oxidation of the methionyl residue in the reaction site of the molecule. This was indicated by findings that activity of the $\alpha-1-\mathrm{PI}$ could be restored by exposure to the reducing agent, dithiothreitol in the presence of methionyl sulfoxide peptide reductase. The amount of activity restored was proportional to the amount of inactive $\alpha$-1-PI present at $52,000 \mathrm{D}$. Oxidation of the $52,000-\mathrm{D} \alpha-1-\mathrm{PI}$ was also revealed by the finding that the inactive molecule was subject to proteolytic cleavage to $47,000 \mathrm{D}$ when exposed to porcine pancreatic elastase, a characteristic of $\alpha$-1-PI with oxidized methionyl residues in the reactive site. Inactivation of the $\alpha-1-P I$ in vivo also resulted from complexing to an active enzyme, shown previously to be neutrophil elastase, and from proteolytic cleavage in vivo, that produced a fragment of $47,000 \mathrm{~mol} \mathrm{wt}$.

In contrast to that in BAL fluids, the $\alpha-1-\mathrm{PI}$ in plasma of patients with respiratory distress syndrome was found to be $>90 \%$ active in 14 of 22 cases and 50$90 \%$ active in 8 cases. This suggested that for the most part, $\alpha-1-P I$ was inactivated after leaving the vessels and entering the lung. The circulating $\alpha-1-P I$ in patients with the respiratory distress syndrome was found to be equally susceptible to oxidative inactivation as $\alpha$-1-PI from normal individuals. It seems improbable therefore that patients develop ARDS because of labile $\alpha$-1-PI inhibitor.
\end{abstract}

Received for publication 11 August 1982 and in revised form 22 November 1982.

\section{INTRODUCTION}

Recent evidence obtained in this laboratory (1) and by Lee et al. (2) has demonstrated in bronchoalveolar lavage (BAL)' fluid of patients with adult respiratory distress syndrome (ARDS), the presence of active neutrophil elastase in a majority of cases. Alpha-1-proteinase inhibitor ( $\alpha$-1-PI, A, AT), also found in a majority of the BAL fluids, was noted to be partly or totally inactive in 9 of 17 cases in which elastase activity was observed, as demonstrated by a lack of binding of radiolabeled trypsin. In five of the cases, a portion of the $\alpha$-1-PI was found by immunoelectrophoresis to be complexed with neutrophilic elastase, accounting for some of the inactivation. In those and other cases, however, free, inactive $\alpha$-1-PI was present.

The inhibition of $\alpha-1$-PI has also been observed in BAL fluids of individuals who smoke (3). The ability of model oxidants and smoke extracts to inactivate the elastase-inhibitory $(\alpha-1-\mathrm{PI})$ capacity of serum was shown (4). Subsequently, stimulated neutrophils or xanthine-xanthine oxidase reactions were observed to inactivate $\alpha-1-\mathrm{PI}$ in serum. The inactivation was attributed to a combination of superoxide anion $\left(\mathrm{O}_{\overline{2}}\right)$ and $\mathrm{H}_{2} \mathrm{O}_{2}$ (5). The product of these reactants, hydroxyl radical $(\cdot \mathrm{OH})$, was suspected of being the major contributor to the inactivation of $\alpha-1-P I$. BAL fluid from rats subjected to smoke inhalation contained inactivated $\alpha-1-P I$, which regained partial activity when dialyzed against reducing agent (6). A twofold reduc-

\footnotetext{
'Abbreviations used in this paper: $\alpha$-1-PI, alpha-1-proteinase inhibitor; ARDS, adult respiratory distress syndrome; BAL, bronchoalveolar lavage; DTT, dithiothreitol; MS reductase, methionyl sulfoxide-peptide reductase; NE, neutrophil elastase; PPE, porcine pancreatic elastase; SDS-PAGE, sodium dodecyl sulfate-polyacrylamide gel electrophoresis; Suc $\mathrm{NCl}, \mathrm{N}$-chlorosuccinimide.
} 
tion in the activity of $\alpha-1-P I$ occurs in BAL-fluid of smoking individuals (7). Thus it appears that $\alpha-1-P I$ is highly susceptible to inactivation by oxidants. The inactivation may involve oxidation of a methionyl residue located in position $\mathrm{Pl}$ of the reaction site of the molecule (8). When this methionyl residue is oxidized to methionyl sulfoxide, the $\alpha-1-P I$ no longer reacts with human neutrophil elastase (9). This oxidant-inactivated $\alpha-1-$ PI retains its native molecular weight.

By contrast, $\alpha-1-P I$ is also subject to inactivation by enzymatic cleavage with thiol proteases (10), by macrophage elastase (11), and by complexing with proteases such as neutrophil elastase (12).

In order to determine the mechanism of inactivation of the $\alpha-1-P I$ in BAL fluids of patients with ARDS, we have examined the $\alpha-1-P I$ in these fluids and in plasma samples taken simultaneously. We reasoned that if oxidation of the $\alpha-1-\mathrm{PI}$ could be demonstrated, this would serve as an indicator of oxidative reactions occurring in the lung and reveal the presence of a process of pathogenic importance.

\section{METHODS}

\section{Materials}

Study population and preparation of BAL samples. The ARDS patients studied were the same as those listed in a previous publication (1). The BAL fluids were stored 1-18 mo at $-70^{\circ}$ and thawed one to three times before use. Storage and thawing up to three times did not affect the results. The fluids were never subject to concentration. The bronchoscopic methods and preparation of BAL fluid was the same as that described (1). Briefly, segments of the lower lobe were lavaged with $20 \mathrm{ml}$ of normal saline, $30-50 \%$ of which was recovered. The cells were removed and the samples frozen in plastic vials. Lavage fluids used in the current study were taken during the time of active ARDS.

Chemicals. $\mathrm{N}$-succinyl-(L-ala- $)_{3}-p$-nitroanalide and $\mathrm{N}$ chlorosuccinimide were obtained from Sigma Chemical Co., St. Louis, MO.

Proteins. $\alpha$-1-PI was purified from human plasma by ammonium sulfate precipitation, DEAE-Sephadex chromatography and chromatography on concanavalin A-Sepharose. Removal of residual albumin was accomplished using DEAE-Affigel Blue (Bio-Rad Laboratories, Richmond, CA) as described (13). $\alpha-1-P I$ concentration was determined by radial immunodiffusion assay (14). $\alpha$-1-PI activity was measured by inhibition of porcine pancreatic elastase (PPE) (13). Purified $\alpha-1-P I$, prepared as noted above, served as standard for analyses of both protein concentration and activity of $\alpha-1-P I$ in complex solutions. The purified $\alpha-1-P I$ contained the same specific activity as that in whole plasma (the pancreatic elastase inhibiting capacity of whole plasma is $>90 \%$ inhibited by treatment with anti- $\alpha-1-P I)$. Protein concentration was measured by the method of Lowry (15). Monospecific antibody to $\alpha$-1-PI was prepared in a goat as described (1). The final antibody preparation yielded a single precipitin band with whole plasma that gave a line of coalescence with a precipitin band formed between the anti$\alpha-1-P I$ and the purified inhibitor. Methionyl sulfoxide-peptide reductase (MS reductase) was provided by Dr. Nathan
Brot, Roche Institute of Molecular Biology, Nutley, NJ, through the courtesy of Dr. Aaron Janoff and Dr. Harvey Carp, Department of Pathology, State University of Stony Brook, NY. PPE was obtained from Sigma Chemical Co.

\section{Methods}

Oxidation of $\alpha-1-P I$. Alpha 1-PI in plasma was oxidized according to the method of Johnson and Travis (9) with appropriate increases in the amount of $\mathrm{N}$-chlorosuccinimide (Suc $\mathrm{NCl}$ ) to compensate for the extra protein in the plasma. Normal human plasma at a final concentration of $1 / 2$ was incubated with $1 \mathrm{mg} / \mathrm{ml}$ of Suc $\mathrm{NCl}$ in $0.05 \mathrm{M}$ (final concentration) Tris buffer, $\mathrm{pH} 8.8$, for $30-60 \mathrm{~min}$ at room temperature. Complete oxidation was confirmed by the loss of ability of the plasma to inhibit PPE. The sample was then dialyzed overnight against Tris-buffered saline pH 7.4 to remove excess Suc NCl.

Preparation of insolubilized anti- $\alpha-1-P I$. Goat antiserum to human $\alpha-1-P I$ was twice absorbed with $25 \mathrm{mg} / \mathrm{ml}$ kaolin at $37^{\circ} \mathrm{C}$ for $30 \mathrm{~min}$. The absorbed antiserum was then brought to $30 \%$ saturation with solid ammonium sulfate at $4^{\circ} \mathrm{C}$ and allowed to precipitate overnight. The precipitate was centrifuged and washed with $45 \%$ saturated ammonium sulfate. The final precipitate was resuspended in, and dialyzed against phosphate-buffered saline, $\mathrm{pH}$ 7.0. This preparation was then passed over a column of Sepharose 4B to which purified $\alpha-1-P I$ had been covalently coupled. When the column was saturated with antibody, it was washed extensively with phosphate-buffered saline to which $1 \mathrm{M} \mathrm{NaCl}$ had been added. Elution of the immunopurified antibody was effected with $5 \mathrm{M}$ guanidine- $\mathrm{HCl}$. Eluted fractions were dialyzed immediately against phosphate-buffered saline to remove the guanidine. The immunopurified antibody was covalently coupled to cyanogen bromide-activated Sepharose $4 \mathrm{~B}$ beads ( $4.3 \mathrm{mg}$ antibody/g beads).

Isolation of $\alpha-1-P I$ from plasma and lavage fuids. Plasma or lavage samples (+ added PPE, see text) were diluted to contain $92 \mu \mathrm{g} / \mathrm{ml}$ of $\alpha-1-P I$. 25- $\mu$ l samples were mixed with $30 \mu \mathrm{l}$ of a slurry of Sepharose 4B containing covalently coupled anti- $\alpha-1-P I$ and incubated at room temperature for $60 \mathrm{~min}$. The beads were centrifuged and washed four times with $50 \mu \mathrm{l}$ of $1 \mathrm{M} \mathrm{NaCl}$ in phosphate-buffered saline and once with $50 \mu \mathrm{l}$ Tris-buffered saline (no added salt). The washed beads were eluted with $15 \mu$ l of $10 \%$ SDS for $3 \mathrm{~min}$ at $100^{\circ} \mathrm{C}$. After centrifugation of the beads, supernatant samples were analyzed by electrophoresis in polyacrylamide gels containing sodium dodecyl sulfate (SDS). Protein bands were revealed by Coomassie Blue stain.

Electrophoresis of proteins. Polyacrylamide gel electrophoresis in sodium dodecyl sulfate (SDS-PAGE) was performed according to the method of Laemmli (16) using a slab gel system. Acrylamide concentrations of 8 or $10 \%$ were used. Gels were fixed and stained using $0.1 \%$ Coomassie Brilliant Blue R-250.

Treatment of lavage fluids with porcine pancreatic elastase. Selected lavage samples were diluted with Tris-buffered saline to contain $115 \mu \mathrm{g} \alpha-1-\mathrm{PI} / \mathrm{ml}$. 20- $\mu$ l aliquots were incubated with $0.5 \mu \mathrm{g} / 5 \mu \mathrm{l}$ of PPE in Tris buffer, $\mathrm{pH} 7.5$ for $30 \mathrm{~min}$ at room temperature prior to isolation of the $\alpha-1-P I$ by immunoabsorption and SDS-PAGE analysis of the $\alpha-1-\mathrm{PI}$.

\section{RESULTS}

Activity of $\alpha-1-P I$ in lavage fluids of patients with $A R D S$. BAL fluids from 24 patients, obtained during 
the course of illness, were examined for their capacity to inhibit PPE. The quantity of $\alpha-1-P I$ activity was obtained by comparison of the inhibitory activity of lavage fluid with that of known quantities of purified $\alpha-1$-PI. The total amount of $\alpha$-1-PI present in the BAL fluid was obtained by quantitative immunodiffusion assays. The data, presented in Fig. 1 , reveal that inhibition of $\alpha-1-P I$ activity was observed in the BAL fluids. In each case, the amount of activity was diminished in comparison with the amount of $\alpha$-1-PI present. 26 of a total of 47 fluids containing $\alpha-1-\mathrm{PI}$ protein, were completely inactive. A poor correlation was found between the total concentration of $\alpha-1-\mathrm{PI}$ and the amount of activity measured. In addition, no correlation was found between the numbers of leukocytes in the BAL fluids and the amount of activity of $\alpha$-1-PI. Continued incubation of BAL fluids with PPE over a period of $60 \mathrm{~min}$ at $37^{\circ}$ failed to elicit inhibition of the elastase.

Structural analysis of inactive $\alpha-1-P I$ in $B A L$ fluids. Certain proteolytic enzymes, including elastase, combine firmly with $\alpha-1-P I$ at the time of inhibition. Under conditions where the inhibited enzyme is dissociated from the $\alpha-1-\mathrm{PI}$, the $\alpha-1-\mathrm{PI}$ may be shown to have undergone proteolytic cleavage. With other enzymes, e.g., papain, the $\alpha-1-P I$ is cleaved and inactivated without strong complex formation with the

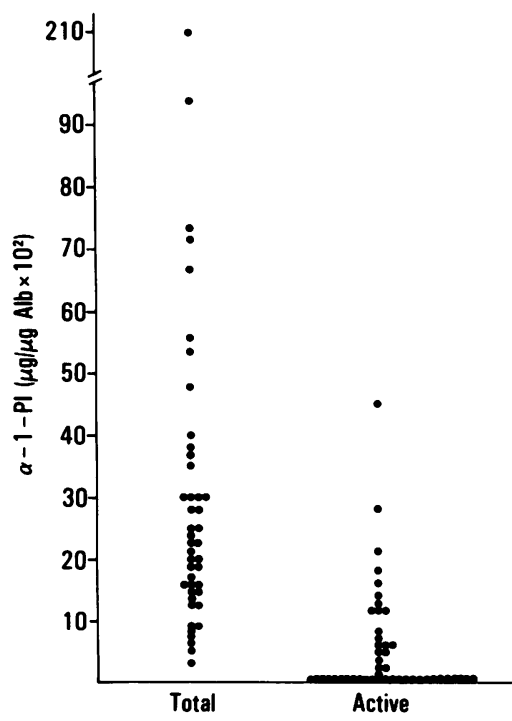

Figure 1 Total and active $\alpha-1-P I$ in BAL fluid of patients with the respiratory distress syndrome. Each dot represents a determination of a single fluid. In several patients, BAL fluids were taken at daily intervals. No more than three fluids were included from a single patient. The concentration of $\alpha-1-P I$ was divided by the concentration of albumin in the fluid to correct for dilution resulting from the saline used for lavage. enzyme. We therefore examined the inactive $\alpha-1-P I$ in BAL fluid for evidence of proteolytic cleavage or combination with enzyme using electrophoresis in SDS-PAGE and immunoelectrophoresis. To examine the $\alpha-1-P I$ in SDS-PAGE, the $\alpha-1-P I$ in BAL fluids was first isolated by immunoaffinity absorption on Sepharose beads to which anti- $\alpha-1-P I$ was covalently coupled (see Methods). Analysis of BAL fluid from eight cases of ARDS that contained sufficient concentrations of $\alpha-1$-PI to allow observation revealed single protein bands at $52,000 \mathrm{~mol}$ wt. In two cases in which the $\alpha$-1-PI was $>98 \%$ inactive, two protein bands were observed, one of $47,000 \mathrm{~mol} w \mathrm{t}$ and another of $\sim 80,000 \mathrm{~mol} w \mathrm{w}$. In each of these cases, neutrophil elastase antigen was observed immunologically in the BAL fluid, and, as noted below, complexes of $\alpha$-1-PI and elastase could be demonstrated by immunologic techniques.

To study the electrophoretic behavior of the inactive $\alpha-1-P I$, immunoelectrophoretic analyses were performed. In each case, $\alpha-1-P I$ was present in the $\alpha$ region, migrating coincidentally with active, purified $\alpha-1-P I$ and with $\alpha-1-P I$ in normal plasma. In 5 of the 24 cases, $\alpha$-1-PI was also observed in the $\beta$-region of the immunoelectrophoretic pattern in the same position as elastase protein published previously (1).

Evidence of oxidation of $\alpha-1-P I$ in BAL fluids. Direct chemical analysis of the metastable methionylsulfoxide in the inactive site of $\alpha-1-P I$ is not readily performed on the relatively small amount of $\alpha-1-P I$ obtainable in the BAL fluids. However, by indirect techniques, evidence of oxidation of the methionyl residue is possible. In addition, by use of MS reductase together with a reducing agent, it is possible to reactivate oxidized $\alpha-1-\mathrm{PI}(17)$. This technique was applied to inactive $\alpha-1-\mathrm{PI}$ of BAL fluids.

Cleavage of $\alpha-1-P I$ in BAL fluids into a 47,000-mol wt fragment by PPE. Johnson and Travis (9) have demonstrated that oxidized $\alpha-1$-PI but not native $\alpha-1$ PI undergoes cleavage by PPE to yield a 47,000-mol wt fragment. To determine by this method if $\alpha-1-P I$ in BAL fluids had undergone oxidative inactivation, BAL fluids containing $\alpha-1-P I$ were exposed to PPE and the $\alpha-1-P I$ then isolated by immunoabsorption prior to analysis in SDS-PAGE (Methods). The results were compared to the action of PPE on Suc NCl-oxidized (and fully inactivated) $\alpha-1-P I$ in normal human plasma and on native $\alpha$-1-PI in normal plasma. BAL fluid from seven patients with ARDS were examined, containing $\alpha$-1-PI with varying degrees of inactivation as shown in Table I. The protein bands containing $\alpha-1-P I$ are shown in the table, and the typical appearance of the bands, in Fig. 2. As shown, when the $\alpha$-1-PI in the fluid was $\approx 50 \%$ active, the band of $52,000 \mathrm{~mol}$ wt was cleaved to form a protein of $47,000 \mathrm{~mol} \mathrm{wt}$. In cases 
TABLE I

Molecular Weight of $\alpha-1-P I$ Peptides in BAL Fluid of Patients with ARDS. Effect of Treatment with PPE

\begin{tabular}{|c|c|c|c|}
\hline \multirow[b]{2}{*}{ Patient } & \multirow{2}{*}{$\begin{array}{c}\alpha-1-\mathbf{P I} \\
\text { Activity }\end{array}$} & \multicolumn{2}{|c|}{ Mol wt of $\alpha-1-\mathrm{PI}$ peptides $\left(\times 10^{-9}\right)$} \\
\hline & & No elastase & Added elastase \\
\hline & $\begin{array}{c}\% \\
\text { active }\end{array}$ & & \\
\hline 187 & 0 & $52,47,80$ & 47,80 \\
\hline 305 & 10 & 52 & 47,80 \\
\hline 160 & 2 & 47,80 & 47,80 \\
\hline 121 & 44 & 52 & 47,80 \\
\hline 102 & 52 & 52 & 47,80 \\
\hline 292 & 75 & 52 & 52,80 \\
\hline 274 & 80 & 52 & 52,80 \\
\hline Control $\alpha-1-\mathrm{PI}$ & 100 & 52 & 52,80 \\
\hline Oxidized $^{\bullet} \alpha-1-$ PI & 0 & 52 & 47 \\
\hline
\end{tabular}

- Oxidized with Suc NCl.

where the activity of the $\alpha-1-P I$ was $<10 \%$ of the total $\alpha-1-P I$ present in the fluid, treatment with PPE resulted in almost complete conversion to the 47,000 mol wt fragment. In cases 102 and 121 where $~ 50 \%$ of the $\alpha-1-\mathrm{PI}$ was active, the PPE treatment yielded a band of $\sim 80,000 \mathrm{~mol}$ wt, probably representing an $\alpha$-1-PI-elastase complex. In cases 274 and 292 in which

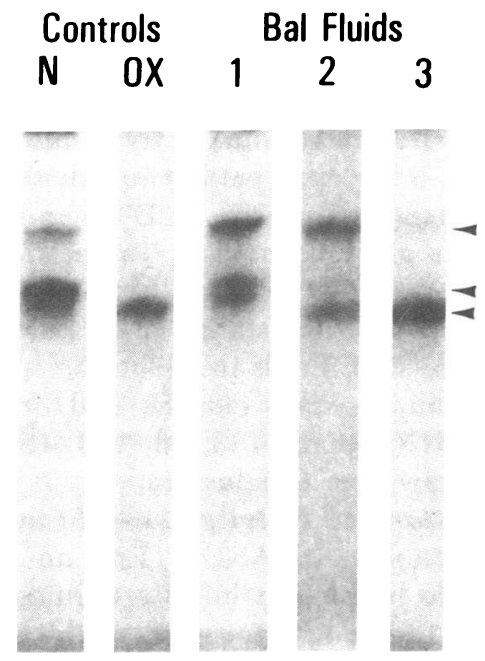

Figure 2 Acrylamide electrophoresis of $\alpha$-1-PI to demonstrate cleavage of oxidized $\alpha-1-P I$ by PPE. Purified human $\alpha-1-P I$, either native (N) or Suc $\mathrm{NCl}$ oxidized (OX), was exposed to PPE. In the right-hand three lanes, $\alpha-1-P I$ in BAL fluids from three patients after PPE treatment is illustrated. $1=80 \%$ active (patient 274 ); $2=44 \%$ active (patient 121 ) $3=$ inactive $\alpha-1$-PI (patient 187). Coomassie Blue stains. Arrows signify: upper $=80,000 \mathrm{D}$; middle $=52,000$; lower $=47,000$.
$>75 \%$ of the $\alpha-1-\mathrm{PI}$ was active, no $47,000-\mathrm{mol}$ wt band appeared, but an $80,000-\mathrm{mol}$ wt band was formed. In these last cases, it is possible that the PPE was complexed and inactivated entirely by the native $\alpha-1-P I$.

Recovery of $\alpha-1-P I$ activity in BAL fluid of ARDS patients by exposure to reductants. Initial experiments were conducted to determine if the activity of $\alpha-1-\mathrm{PI}$, inactivated by oxidation by Suc $\mathrm{NCl}$, could be reconstituted by reduction with dithiothreitol (DTT) in the presence of MS reductase. Accordingly, normal human plasma was treated with Suc $\mathrm{NCl}$ so that the plasma no longer inhibited PPE. After dialysis to remove excess Suc $\mathrm{NCl}$, the plasma was diluted in saline and an incubation mixture containing the following was prepared: $38.3 \mu \mathrm{g} / \mathrm{ml}$ oxidized $\alpha$-1-PI, $13 \mathrm{mM}$ DTT, $13 \mathrm{mM} \mathrm{MgCl} 2,33.3 \mathrm{mM}$ Tris, $\mathrm{pH} \mathrm{7.4,} \mathrm{and} 106.7$ $\mu \mathrm{g} / \mathrm{ml}$ MS reductase (method of Abrams et al. [17]). Incubation was carried out at $37^{\circ} \mathrm{C}$ for 1,120 , and 390 min. The reaction was stopped by cooling on ice, and then $\alpha-1-P I$ activity assessed with PPE as noted in Methods. The oxidized plasma yielded no $\alpha$-1-PI inhibitory activity, while reduction for the three incubation times given produced recoveries of 2.5, 14.6, and $14.0 \mu \mathrm{g} / \mathrm{ml} \alpha-1-P I$ activity, or $\sim 38 \%$ of the available $\alpha$-1-PI. When purified $\alpha$-1-PI was oxidized and then incubated with DTT and MS reductase as above, full activity was restored in $2 \mathrm{~h}$.

The BAL fluids from patients with ARDS were then examined for potential restoration of $\alpha$-1-PI activity. BAL fluids were chosen that contained 25 to $130 \mu \mathrm{g}$ total $\alpha-1-\mathrm{PI} / \mathrm{ml}$ with varying degrees of inactivity. The BAL fluids were then exposed to DTT and MS reductase for $2 \mathrm{~h}$ as noted above and the PPE-inhibitory activity assessed. The $\alpha-1-P I$ activity before and after exposure to DTT and MS reductase for each fluid is presented in Fig. 3. The data are arranged so that those BAL fluids in which there is greatest restoration of activity appear in the left-hand columns. As can be seen, substantial restoration of PPE-inhibitory activity developed. Control samples of each BAL fluid incubated as above but without MS reductase showed no appreciable changes in PPE-inhibitory activity over a $2-h$ period. Since the PPE inhibitory capacity of BAL fluids is blocked by immunopurified antibody to $\alpha-1-$ PI, and ${ }^{125}$ I-trypsin is bound only by $\alpha-1-P I$ in the BAL fluid (1), we presume that the restored activity was that of $\alpha$-1-PI.

It was of note that in several BAL fluids, almost total $\alpha$-1-PI activity was restored. In others, by contrast, incomplete, or in one case (patient 160 ) no restoration occurred even though there was an abundance of $\alpha$ 1-PI in the fluid. To determine the cause of this, the $\alpha-1-P I$ of the BAL fluids was examined by SDS-PAGE in the absence of any prior treatment of the BAL fluid. The $\alpha$-1-PI was isolated by immunoaffinity-isolation 


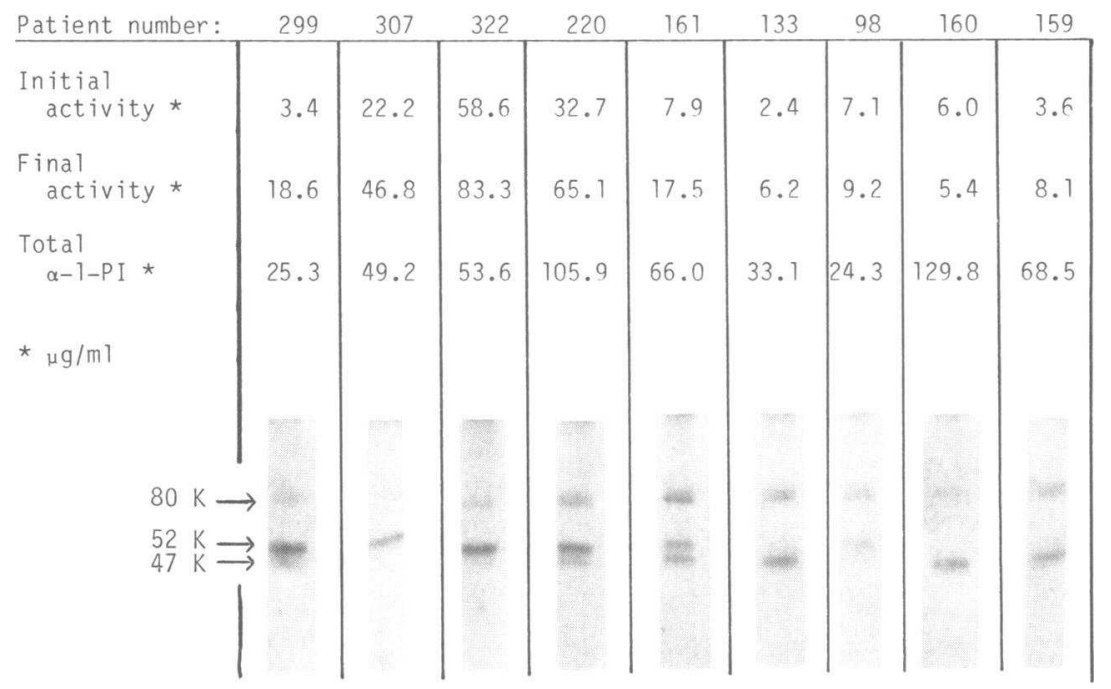

FIGURE 3 Reactivation of $\alpha$-1-PI from BAL fluid of patients with ARDS by exposure to DTT in the presence of MS reductase. The total $\alpha$-1-PI levels were obtained by immunodiffusion methods, and activity of the $\alpha-1-P I$, with or without exposure to DTT, and reductase, by its capacity to inactivate PPE. Acrylamide electrophoresis, shown at the bottom, was performed on $\alpha-1-P I$ isolated from untreated BAL fluids after immunoaffinity isolation of the $\alpha-1-\mathrm{PI}$.

on Sepharose 4B beads upon which anti- $\alpha$-1-PI was covalently coupled. After washing, the $\alpha-1-P I$ was eluted with SDS and its position in SDS-PAGE determined. The results are presented in the bottom portion of Fig. 3. It can be seen that when restoration of activity was limited, as in samples to the right of the figure that $\alpha-1-P I$ was found to be present in cleaved, 47,000-mol wt form or in the complexed, $80,000-\mathrm{mol}$ wt form. Fluids containing the 80,000 -mol wt form of $\alpha-1-P I$ were observed using immunodiffusion assays to contain neutrophil elastase as well (data not shown). This large molecular weight form presumably represented the complex of neutrophil elastase and $\alpha-1-P I$ observed previously (1). When restoration of activity of $>10 \mu \mathrm{g} \alpha-1-\mathrm{PI} / \mathrm{ml}$ was observed, there was invariably $\alpha$-1-PI present at 52,000 mol wt in SDS-PAGE. Since in most cases, some $\alpha-1-\mathrm{PI}$ activity was found without reduction, a portion of the $\alpha$-1-PI at 52,000 mol wt represented active, unoxidized protein. It is of note that in patient $307,44 \%$ of the $\alpha-1-P I$ present was active initially, and $95 \%$ of the total $\alpha$-1-PI was active after reduction. The SDS-PAGE pattern of the untreated $\alpha-1-\mathrm{PI}$ in accordance with these data, revealed all the $\alpha$-1-PI to be at $52,000 \mathrm{~mol} \mathrm{wt}$. We would have anticipated the presence of protein at $52,000 \mathrm{~mol} \mathrm{wt}$ in patients 133 and 159. Presumably the trace amount of active and oxidized $\alpha-1-P I$ in that position in the gels was lost in the immunopurification procedure. The value of total $\alpha-1-P I$ in patient 322 was low and may represent an error in quantitation.

Oxidants could be derived from various sources such as leukocytes, stimulated to release superoxide anion, arachidonate metabolites produced by stimulated leukocytes, and from superoxide anion generated during the reduction of molecular oxygen in the presence of high levels of inspired oxygen. To examine the last possibility, we sought to determine if a correlation existed between the concentration of inspired oxygen and the amount of $\alpha-1-P I$ inactivated presumably by oxidation. We used the values of total $\alpha-1-P I$ and recovered $\alpha$-1-PI activity (final activity-initial activity) shown in Fig. 3 for each patient to calculate the percent reactivated by exposure to DTT and MS reductase. The data are presented in Table II. The results suggest that a positive correlation does not exist. There are insufficient data for statistical evaluation. In addition, in those BAL fluids in which the $\alpha$-1-PI was in great part complexed to elastase and/or cleaved to $47,000 \mathrm{~mol}$ wt (patients $133,98,160,159$ ), it is not possible to draw firm conclusions.

Concentration and activity of $\alpha-1-P I$ in circulating plasma of patients with ARDS. Free neutrophil elastase, known to be present in lavage fluids of patients with ARDS $(1,2)$ could injure the lung parenchyma in this disease. Control of the elastase would be compromised if individuals were deficient in $\alpha-1-\mathrm{PI}$, had inactive $\alpha-1-\mathrm{PI}$, or $\alpha-1-\mathrm{PI}$ that was readily inactivated by oxidation. $\alpha-1-\mathrm{PI}$ in plasma from ARDS patients was examined during the time of illness to test these possibilities.

The concentration and activity of $\alpha-1-P I$ in the circulating plasma was found to be elevated in all patients 
TABLE II

Relationship of the Concentration of Oxygen in the Inspired Air with Inactivation of $\alpha-1-P I$ in BAL Fluid

\begin{tabular}{lccccccccc}
\hline \multicolumn{1}{c}{ Patient . . } & 299 & 307 & 322 & 220 & 161 & 133 & 98 & 160 & 159 \\
\hline $\mathrm{FiO}_{2}{ }^{\circ}$ & 0.3 & 0.4 & 0.9 & 0.45 & 0.3 & 0.6 & 0.21 & 0.6 & 0.5 \\
$\begin{array}{l}\text { Days FiO } \\
\quad>0.50 \ddagger\end{array}$ & 1 & 0 & 1 & 0 & 0 & 18 & 0 & 9 & 4 \\
$\begin{array}{c}\text { Reactivated } \\
\quad \alpha-1-P I, \% \S\end{array}$ & 60.1 & 50.0 & $<46$ & 30.6 & 14.5 & 11.4 & 8.6 & 0 & 6.6 \\
\hline
\end{tabular}

- Fractions of oxygen in inspired air $(1.0=100 \%$ oxygen $)$ on day BAL fluid sample obtained.

$\ddagger$ Consecutive days prior to $\mathrm{BAL}$ sample at which $\mathrm{FiO}_{2}>0.5$.

$\S \alpha-1-\mathrm{PI}$ reactivated by exposure to DTT and MS reductase (Fig. 3) as a function of total $\alpha$-1-PI present $\times 100$.

within the first $4 \mathrm{~d}$ of developing ARDS (Fig. 4). In 14 of the 22 plasmas examined, $<10 \%$ difference between total and active $\alpha-1-P I$ was observed. In the remaining eight cases, $11-50 \%$ of the $\alpha-1-P I$ was inactive. Immunoelectrophoretic analysis of these cases showed $\alpha-1-P I$ only in the $\alpha-1$ region, suggesting it was not associated with enzymes. The level of $\alpha-1-P I$ in the plasma of seven normal individuals was found to be $3.1 \pm 1.5 \mathrm{mg} / \mathrm{ml}$, with $>71 \%$ of the circulating $\alpha$-1-PI active.

Susceptibility of $\alpha-1-P I$ of patients with ARDS to inactivation by oxidation was analyzed by exposing 25 $\mu$ l plasma, diluted $1 / 25$ in Tris-saline, to $1.76 \mu \mathrm{g}$ Suc $\mathrm{NCl}$ for $15 \mathrm{~min}, 22^{\circ} \mathrm{C}$. This amount of Suc $\mathrm{NCl}$ was chosen to inactivate $\sim 50 \%$ of the $\alpha-1-\mathrm{PI}$ in normal plasma. Plasma from 12 cases of ARDS and 8 normal individuals was tested. After exposure, activity of $\alpha$ 1-PI in the plasma was tested for its ability to inactivate PPE as noted in Methods. The results are shown in Fig. 5. As shown, there was no detectable difference in the inactivation of $\alpha-1-P I$ in ARDS and normal plasmas. When tested for inactivation each $15 \mathrm{~min}$ over a $60-\mathrm{min}$ period, the rate of inactivation of $\alpha-1-\mathrm{PI}$ in six ARDS and four normal plasmas was found to be $0.82 \pm 0.07 \%$ of the total $\alpha-1-P I$ present $/ \mathrm{min}$ in the

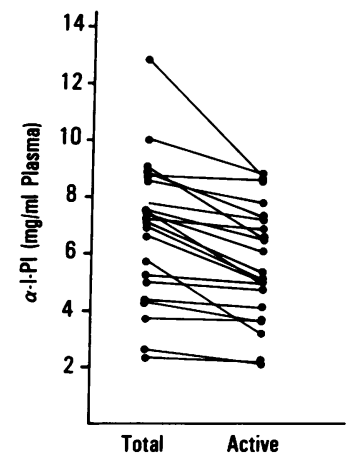

FIGURE 4 Total concentration and activity of $\alpha-1-P I$ in plasma of ARDS patients taken during the illness. The lines connect individual points of the same patient.
ARDS plasmas, and $0.69 \pm 0.09 \% / \mathrm{min}$ in normal plasmas. The inactivation of $\alpha-1-\mathrm{PI}$ by Suc $\mathrm{NCl}$ was linear over this time period. Thus little difference in the total amount of oxidative inactivation of $\alpha-1-\mathrm{PI}$ or the rate of inactivation was noted.

\section{DISCUSSION}

The present study provides evidence that $\alpha-1-P I$, becomes inactivated in the lungs of patients with ARDS. Inactivation of the $\alpha-1-P I$ apparently resulted from three causes: oxidation, complex formation with neutrophil elastase (NE) and proteolytic cleavage of the $\alpha-1-P I$. In 15 of 17 cases analyzed, all or a portion of the $\alpha-1-P I$ was found at $52,000 \mathrm{~mol} \mathrm{wt}$, either totally or partially inactive. As will be discussed below, evidence indicated that this inactive $\alpha$-1-PI had undergone oxidation. In 9 of 17 cases analyzed, a portion of the $\alpha-1-P I$ was found at $80,000 \mathrm{~mol}$ wt in SDSPAGE. Our previous observations (1) indicated that complexes of $\alpha-1-\mathrm{PI}$ and $\mathrm{NE}$ existed in these BAL

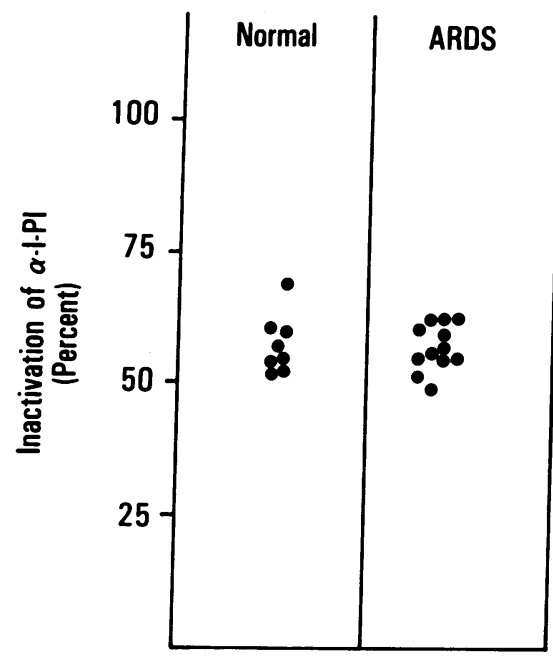

Figure 5 Inactivation of $\alpha-1-P I$ in plasma of normal individuals and patients with ARDS with Suc NCl. 
fluids, findings that in all probability account for the present $80,000-\mathrm{mol}$ wt form of the inhibitor protein. In 7 of the 17 cases, a portion of the $\alpha$-1-PI was detected at $47,000 \mathrm{~mol} \mathrm{wt}$.

The present evidence suggested that the inactive $\alpha-1-P I$ at $52,000 \mathrm{~mol}$ wt had undergone a reaction of oxidation. Both indirect and direct evidence supported this conclusion. Oxidized $\alpha-1-\mathrm{PI}$ is cleaved by PPE to yield a major fragment of $47,000 \mathrm{~mol} \mathrm{wt}(8,9)$. We confirmed this observation using $\alpha-1-\mathrm{PI}$ isolated from plasma oxidized in vitro with Suc $\mathrm{NCl}$. We then observed that inactive $\alpha-1-\mathrm{PI}$, isolated from the BAL fluid of patients with ARDS, also could be cleaved with PPE to yield the $47,000-\mathrm{mol}$ wt fragment.

Using a more direct approach to the question, it was possible to reactivate the $\alpha$-1-PI in lavage fluid by exposure to DTT in the presence of MS reductase. The amount of $\alpha-1-P I$ activity that was recovered by reduction corresponded roughly to the amount of $\alpha-1$ PI of 52,000 mol wt present in the BAL fluid. When $>10 \mu \mathrm{g} / \mathrm{ml} \alpha-1$-PI was reactivated, $\alpha-1-\mathrm{PI}$ at 52,000 mol wt was invariably present. In BAL fluid from some patients, all of the inactive $\alpha-1-\mathrm{PI}$ was converted to the active form by reduction. However, the amount of $\alpha$-1-PI reactivated could not be quantitated in all cases since elastase-complexed or cleaved $(47,000 \mathrm{~mol}$ wt) $\alpha$-1-PI was present in the BAL fluids examined. The presence of 47,000-mol wt $\alpha-1-P I$ in the BAL fluid indicates that oxidative inactivation followed by proteolytic cleavage had occurred in vivo. Thus the relationship of active and activatable $\alpha-1-P I$ to the presence of $52,000-\mathrm{D} \alpha-1-\mathrm{PI}$ in the individual BAL fluids supports the concept of oxidative inactivation of the inhibitor

It is likely that the activity obtained after treatment with reducing agent and MS reductase is that of $\alpha-1$ PI, since $>90 \%$ of the elastase inhibitory capacity of the BAL fluid is attributable to $\alpha-1-P I$ (it is blocked by immunopurified anti- $\alpha-1-P I)$. Unfortunately, the small quantities of $\alpha-1-\mathrm{PI}$ in the individual BAL fluid sample made direct demonstration of elastase binding to the reactivated $\alpha-1-P I$ technically unfeasible.

It is not possible to exclude unequivocally the possibility that inactivation of the $\alpha-1-P I$ had occurred after removal of the BAL fluid from the patient. The finding of fully active $\alpha-1-\mathrm{PI}$ in BAL fluid of patients with other types of pulmonary disease, e.g., with carcinoma, or in the recovery period in ARDS patients, suggests that the lavage procedure is not responsible for inactivation. Leukocytes, a primary source of elastase, were present in such lavage fluids as well. $\alpha-1-P I$ in plasma from patients with ARDS was frequently fully active. Thus the data infer that the $\alpha$-1-PI of $52,000 \mathrm{~mol} w \mathrm{w}$ undergoes oxidative inactivation or complexing with elastase primarily in the pulmonary tissues.
The source of oxidant in the lung is unclear. Stimulated neutrophils have been shown to release oxidants (leukocyte-generated oxidants) sufficient to inactivate $\alpha-1-P I(18-20)$ and suppress the elastase inhibiting capacity of plasma (5). Neutrophil myeloperoxidase, together with $\mathrm{H}_{2} \mathrm{O}_{2}$ and halide inactivate $\alpha$-1-PI, and the reactive-site methionyl residue has been shown to be oxidized (21). In ARDS, NE was detected by activity or antigenicity in $>80 \%$ of ARDS cases (1), suggesting that these cells were either stimulated or underwent lysis. However, the known stimulant of neutrophils, C5a was detected by radioimmune assay in the BAL fluids of four ARDS patients studied. Another source of oxidant is the elevated concentrations of oxygen administered to the patients. All patients received supplemental $\mathrm{O}_{2}$ therapy during the course of their disease. Lung edema, induced by toxic levels of oxygen, may require the presence of neutrophils, as has been demonstrated by experimental animals $(22,23)$, although rabbits, depleted of polymorphs, have been reported to develop pulmonary edema and die in $100 \%$ oxygen in the same time period as untreated rabbits (24). The relative contribution of leukocyte-derived oxidant, and that resulting from inspired oxygen could not be precisely determined. As noted in Table II, however, there was no correlation observed between the amount of $\alpha-1-\mathrm{PI}$ activity recovered by exposure to reducing agents (Fig. 3) and the concentration of inspired oxygen administered to the patient.

The importance of the observation that $\alpha-1-P I$ was inactivated by oxidation in lungs of patients with ARDS, and, by implication, that oxidants are generated in the lungs, lies not only in the inability of the oxidized $\alpha-1-\mathrm{PI}$ to inactivate NE, but also that the oxidants generated in the tissues could contribute to the injury of the lung in ARDS. That oxidants can injure pulmonary tissue has been documented by several investigators. Stimulated human neutrophils induced increased vascular permeability in isolated, perfused rabbit lungs, while stimulated neutrophils of a patient with chronic granulomatous disease, that did not generate oxidants upon stimulation, failed to increase the permeability (25). The generation of free radicals of oxygen in explants of lung has recently been shown to lyse ${ }^{51} \mathrm{Cr}$-labeled cells in the explant (26). In rats, the combination of glucose oxidase, glucose, and lactoperoxidase (or myeloperoxidase) induced acute pulmonary injury marked by increased vascular permeability (27). The injury was inhibited in a dose-dependent manner by catalase. Xanthine and xanthine oxidase also generated acute edema of the lungs and was partially inhibited by superoxide dismutase but not catalase. The data implicate the generation of oxidants in the induction of acute pulmonary injury. Injury of pulmonary tissues following exposure to high levels of oxygen have been attributed to oxidizing free 
radicals of oxygen generated in vivo $(28,29)$. Thus abundant data exist that implicate oxidant injury of pulmonary tissues.

\section{ACKNOWLEDGMENTS}

The authors wish to thank Mrs. Monica Bartlett for preparation of the manuscript, and Mr. Benjamin Bohl for expert technical assistance.

This is publication 2793 from the Department of Immunology, Scripps Clinic and Research Foundation, La Jolla, CA. This work was supported in part by National Institutes of Health grants AI-17354 and University of California, San Diego Subcontract, the Office of Naval Research, and the Council for Tobacco Research.

\section{REFERENCES}

1. McGuire, W. W., R. G. Spragg, A. B. Cohen, and C. G Cochrane. 1982. Studies on the pathogenesis of the adult respiratory distress syndrome. J. Clin. Invest. 69: 543553.

2. Lee, C. T., A. M. Fein, M. Lippmann, H. Holtzman, P. Kimbel, G. Weinbaum. 1981. Elastolytic activity in pulmonary lavage fluid from patients with adult respiratory distress syndrome. N. Engl. J. Med. 304: 192-196.

3. Carp, H., F. Miller, J. R. Hoidal, and A. Janoff. 1982. Potential mechanism of emphysema: $\alpha$ 1-proteinase inhibitor recovered from lungs of cigarette smokers contains oxidized methionine and has decreased elastase inhibitory capacity.

4. Carp, H., and A. Janoff. 1978. Possible mechanisms of emphysema in smokers. In vitro suppression of serum elastase-inhibitory capacity in fresh cigarette smoke and its prevention by antioxidants. Am. Rev. Respir. Dis. 118: 617-621

5. Carp, H., and A. Janoff. 1979. In vitro suppression of serum elastase-inhibitory capacity by reactive oxygen species generated by phagocytosing polymorphonuclear leukocytes. J. Clin. Invest. 63: 793-797.

6. Janoff, A., H. Carp, D. K. Lee, and R. T. Drew. 1979. Cigarette smoke inhalation decreases $\alpha$-1-antitrypsin activity of rat lung. Science (Wash.DC). 206: 1313-1314.

7. Gadek, J. E., G. B. Fills, and R. G. Crystal. 1979. Cigarette smoking induces functional antiprotease deficiency in the lower respiratory tract of humans. Science (Wash. DC). 206: 1315-1316.

8. Johnson, D., and J. Travis. 1978. Structural evidence for methionine at the reaction site of $\alpha$-1-proteinase inhibitor. J. Biol. Chem. 253: 7142-7144.

9. Johnson, D., and J. Travis. 1979. The oxidative inactivation of human $\alpha$-1-proteinase inhibitor. Further evidence for methionine at the reactive center. J. Biol. Chem. 254: 4022-4026.

10. Johnson, D., and J. Travis. 1977. Inactivation of human alpha-1-proteinase inhibitor by thiol proteinases. Biochem. J. 163: 639-641.

11. Banda, M. J., E. J. York, and Z. Werb. 1980. Limited proteolysis by macrophage elastase inactivates human $\alpha$-1-proteinase inhibitor. J. Exp. Med. 152: 1563-1570, 1980.

12. Ohlsson, K. 1978. In Neutral Proteases of Human Polymorphonuclear Leukocytes. K. Havemann, and A. Jan- off, editors. Urban \& Schwarzenberg, Inc., Baltimore. pp. 167-177, 1, 2.

13. Pannell, R., D. Johnson, and J. Travis. 1974. Isolation and properties of human plasma $\alpha$-1-proteinase inhibitor. Biochemistry. 13: 5439-5445.

14. Mancini, G. A., A. O. Carbonas, and J. F. Heremans. 1965. Immunological quantitation of antigen by single radial immunodiffusion. Immunochemistry. 2: 235-254.

15. Lowry, O. H., N. J. Rosebrough, A. J. Farr, and R. J. Randall. 1951. Protein measurement with the Folin phenol reagent. J. Biol. Chem. 193: 265-274.

16. Laemmli, U. K. 1970. Cleavage of structural proteins during the assembly of the head of bacteriophage T4. Nature (Lond.). 227: 680-685.

17. Abrams, W. R., G. Weinbaum, L. Weissbach, H. Weissbach, and N. Brot. 1981. Enzymatic reduction of oxidized $\alpha$-1-proteinase inhibitor restores biologic activity. Proc. Natl. Acad. Sci. USA. 78: 7483-7486.

18. Babior, B. M., R. Kipnes, and J. Curmutte. 1973. Biological defense mechanisms. The production by leukocytes of superoxide, a potential bactericidal agent. $J$. Clin. Invest. 52: 741-744.

19. Goldstein, I. M., D. Roos, H. B. Kaplan, and G. Weissmann. 1975. Complement and immunoglobulins stimulate superoxide production by human leukocytes independently of phagocytosis. J. Clin. Invest. 56: 11551163.

20. Klebanoff, S. J., and H. Rosen. 1978. Ethylene formation by polymorphonuclear leukocytes. Role of myeloperoxidase. J. Exp. Med. 148: 490-506.

21. Matheson, N. R., P. S. Wong, and J. Travis. 1979. Enzymatic inactivation of alpha-1-proteinase inhibitor by neutrophil myeloperoxidase. Biochem. Biophys. Res. Commun. 88: 402-409.

22. Shasby, D. M., S. S. Shasby, C. M. Bowman, R. B. Fox, R. N. Harada, R. M. Tate, and J. E. Repine. 1981. Lung lavage angiotensin converting enzyme concentrations in normal and nitrogen mustard treated rabbits exposed to hyperoxia. Am. Rev. Respir. Dis. 124: 202-203.

23. Fox, R. B., J. R. Hoidal, D. M. Brown, and J. E. Repine. 1981. Pulmonary inflammation due to oxygen toxicity: involvement of chemotactic factors and polymorphonuclear leukocytes. Am. Rev. Respir. Dis. 123: 521-523.

24. Raj, J., and R. Bland. 1982. Neutrophil depletion does not prevent oxygen-induced lung injury in rabbits. 25th Aspen Lung Conference, June, 1982.

25. Shasby, D. M., K. M. Van Benthuysen, R. M. Tate, S. S. Shasby, I. Mc Murty, and J. E. Repine. 1982. Granulocytes mediate acute edematous lung injury in rabbits and in isolated rabbit lungs perfused with phorbol myristate acetate: role of oxygen radicals. Am. Rev. Respir. Dis. 125: 443-447.

26. Martin, W. J., J. E. Gadek, G. W. Hunninghake, and R. G. Crystal. 1981. Oxidant injury of lung parenchymal cells. J. Clin. Invest. 68: 1277-1294.

27. Johnson, K. J., J. C. Fantone III, J. Kaplan, and P. A. Ward. 1981. In vivo damage of rats lungs by oxygen metabolites. J. Clin. Invest. 67: 983-993.

28. Saltzman, H. A., and I. Fridovich. 1973. Oxygen toxicity. Introduction to a protective enzyme: Superoxide dismutase. Circulation. 48: 921-923.

29. Rister, M., and R. L. Baehner. 1976. The alteration of superoxide dismutase, catalase, glutathione peroxidase, $\mathrm{NADPH}$, cytochrome $c$ reductase in guinea pig polymorphonuclear leukocytes and alveolar macrophages during hyperoxia. J. Clin. Invest. 58: 1174-1184. 